\title{
Solving Integral Representations Problems for the Stationary Schrödinger Equation
}

\author{
Yudong Ren \\ Department of Mathematics and Information Science, Henan University of Economics and Law, Zhengzhou 450002, China \\ Correspondence should be addressed to Yudong Ren; yudong83@163.com
}

Received 24 April 2013; Accepted 9 June 2013

Academic Editor: Pavel Kurasov

Copyright ( 2013 Yudong Ren. This is an open access article distributed under the Creative Commons Attribution License, which permits unrestricted use, distribution, and reproduction in any medium, provided the original work is properly cited.

When solutions of the stationary Schrödinger equation in a half-space belong to the weighted Lebesgue classes, we give integral representations of them, which imply known representation theorems of classical harmonic functions in a half-space.

\section{Introduction and Results}

Let $\mathbf{R}$ and $\mathbf{R}_{+}$be the sets of all real numbers and of all positive real numbers, respectively. Let $\mathbf{R}^{n}(n \geq 2)$ denote the $n$ dimensional Euclidean space with points $x=\left(x^{\prime}, x_{n}\right)$, where $x^{\prime}=\left(x_{1}, x_{2}, \ldots, x_{n-1}\right) \in \mathbf{R}^{n-1}$ and $x_{n} \in \mathbf{R}$. The unit sphere and the upper half unit sphere in $\mathbf{R}^{n}$ are denoted by $\mathbf{S}^{n-1}$ and $\mathbf{S}_{+}^{n-1}$, respectively. The boundary and closure of an open set $D$ of $\mathbf{R}^{n}$ are denoted by $\partial D$ and $\bar{D}$, respectively. The upper half space is the set $H=\left\{\left(x^{\prime}, x_{n}\right) \in \mathbf{R}^{n} ; x_{n}>0\right\}$, whose boundary is $\partial H$.

We identify $\mathbf{R}^{n}$ with $\mathbf{R}^{n-1} \times \mathbf{R}$ and $\mathbf{R}^{n-1}$ with $\mathbf{R}^{n-1} \times\{0\}$, writing typical points $x, y \in \mathbf{R}^{n}$ as $x=\left(x^{\prime}, x_{n}\right), y=\left(y^{\prime}, y_{n}\right)$, where $y^{\prime}=\left(y_{1}, y_{2}, \ldots, y_{n-1}\right) \in \mathbf{R}^{n-1}$ and putting

$$
\begin{array}{cc}
x \cdot y=\sum_{j=1}^{n} x_{j} y_{j}, & |x|=\sqrt{x \cdot x}, \\
\Theta=\frac{x}{|x|}, & \Phi=\frac{y}{|y|} .
\end{array}
$$

For $x \in \mathbf{R}^{n}$ and $r>0$, let $B(x, r)$ denote the open ball with center at $x$ and radius $r(>0)$ in $\mathbf{R}^{n} . S_{r}=\partial B(O, r)$, where $O$ is the origin of $\mathbf{R}^{n}$. For a set $E, E \subset \mathbf{R}_{+} \cup\{0\}$, we denote $\{x \in H ;|x| \in E\}$ and $\{x \in \partial H ;|x| \in E\}$ by $H E$ and $\partial H E$, respectively. By $H r$ we denote $H \cap S r$. We denote by $d S_{r}$ the $(n-1)$-dimensional volume elements induced by the Euclidean metric on $S_{r}$.
Let $\mathscr{A}_{b}$ denote the class of nonnegative radial potentials $b(x)$, that is, $0 \leq b(x)=b(|x|), x \in H$, such that $b \in L_{\text {loc }}^{a}(H)$ with some $a>n / 2$ if $n \geq 4$ and with $a=2$ if $n=2$ or $n=3$.

This paper is devoted to the stationary Schrödinger equation:

$$
\operatorname{SSE}_{b} h(x)=-\Delta h(x)+b(x) h(x)=0
$$

where $x \in H, \Delta$ is the Laplace operator and $b \in \mathscr{A}_{b}$. Note that solutions of (2) are (classical) harmonic functions in the case $b=0$. Under these assumptions the operator $S S E_{b}$ can be extended in the usual way from the space $C_{0}^{\infty}(H)$ to an essentially self-adjoint operator on $L^{2}(H)$ (see [1]). We will denote it by $S S E_{b}$ as well. This last one has a Green function $G_{b}(x, y)$. Here $G_{b}(x, y)$ is positive on $H$ and its inner normal derivative $\partial G_{b}(x, y) / \partial n\left(y^{\prime}\right) \geq 0$. We denote this derivative by $P_{b}\left(x, y^{\prime}\right)$, which is called the Poisson $b$-kernel with respect to $H$. If $G(x, y)$ and $P\left(x, y^{\prime}\right)$ are denoted by the Green function and Poisson kernel of the Laplace operator in $H$, respectively, then

$$
P\left(x, y^{\prime}\right)=-\left.\frac{\partial G(x, y)}{\partial y_{n}}\right|_{y_{n}=0}=\frac{2 x_{n}}{w_{n}} \frac{1}{\left|x-y^{\prime}\right|^{n}},
$$

where $x=\left(x^{\prime}, x_{n}\right), y=\left(y^{\prime}, y_{n}\right)$ and $w_{n}$ is the area of the unit sphere in $\mathbf{R}^{n}$. 
Let $g$ be a continuous function on $\partial H$. We say that $h$ is a solution of the Dirichlet problem for the stationary Schrödinger operator $S S E_{b}$ on $H$ with $g$, if

$$
\begin{gathered}
h \in C^{2}(H) \cap C^{0}(\bar{H}), \\
S S E_{b} h=0 \quad \text { in } H, \\
h=g \text { on } \partial H .
\end{gathered}
$$

Note that $h$ is a solution of the classical Dirichlet problem for the Laplace operator $\Delta$ on $H$ with $g$ in the case $b=0$.

Let $\Lambda$ be a Laplace-Beltrami operator (spherical part of the Laplace) on the unit sphere. It is known (see, e.g., [2, page 41]) that the eigenvalue problem

$$
\begin{aligned}
\Lambda \varphi(\Theta)+\tau \varphi(\Theta)=0 & \Theta \in \mathbf{S}_{+}^{n-1} \\
\varphi(\Theta)=0 & \Theta \in \partial \mathbf{S}_{+}^{n-1}
\end{aligned}
$$

has the eigenvalues $\tau_{j}=j(j+n-2)$, where $j=0,1,2 \ldots$. Corresponding eigenfunctions are denoted by $\varphi_{j v}(1 \leq$ $\left.v \leq v_{j}\right)$, where $v_{j}$ is the multiplicity of $\tau_{j}$. We norm the eigenfunctions in $L^{2}\left(\mathbf{S}_{+}^{n-1}\right)$ and $\varphi_{1}=\varphi_{11}>0$.

Let $P_{j}(r)$ and $Q_{j}(r)$ stand, respectively, for the increasing and nonincreasing, as $r \rightarrow+\infty$, solutions of the equation:

$$
\begin{aligned}
& -T^{\prime \prime}(r)-\frac{n-1}{r} T^{\prime}(r) \\
& \quad+\left(\frac{\tau_{j}}{r^{2}}+b(r)\right) T(r)=0, \quad 0<r<\infty,
\end{aligned}
$$

normalized under the condition $P_{j}(1)=Q_{j}(1)=1$.

We shall also consider the class $\mathscr{B}_{b}$, consisting of the potentials $b \in \mathscr{A}_{b}$ such that there exists a finite limit $\lim _{r \rightarrow \infty} r^{2} b(r)=s \in[0, \infty)$; moreover, $r^{-1}\left|r^{2} b(r)-s\right| \epsilon$ $L(1, \infty)$. If $b \in \mathscr{B}_{b}$, then solutions of (2) are continuous (see [3]).

In the rest of paper, we assume that $b \in \mathscr{B}_{b}$ and we shall suppress this assumption for simplicity. Further, we use the standard notations $u^{+}=\max \{u, 0\}, u^{-}=-\min \{u, 0\}$, and $[d]$ is the integer part of $d$ and $d=[d]+\{d\}$, where $d$ is a positive real number.

Denote

$$
\lambda_{j, s}^{ \pm}=\frac{2-n \pm \sqrt{(n-2)^{2}+4\left(s+\tau_{j}\right)}}{2} \quad(j=0,1,2,3, \ldots) .
$$

Remark 1. $\lambda_{j, 0}^{+}=j$ in the case $b=0$, where $j=0,1,2,3, \ldots$.

It is known (see [4]) that in the case under consideration the solutions to (6) have the asymptotics

$$
P_{j}(r) \sim d_{1} r^{\lambda_{j, s}^{+}}, \quad Q_{j}(r) \sim d_{2} r^{\lambda_{j, s}^{-}}, \quad \text { as } \quad r \longrightarrow \infty,
$$

where $d_{1}$ and $d_{2}$ are some positive constants.
If $b \in \mathscr{A}_{b}$, it is known that the following expansion for the Green function $G_{b}(x, y)$ (see [5, Chapter 11], [6]):

$$
\begin{aligned}
G_{b}(x, y)=\sum_{j=0}^{\infty} & \frac{1}{\chi^{\prime}(1)} P_{j}(\min (|x|,|y|)) \\
& \times Q_{j}(\max (|x|,|y|))\left(\sum_{v=1}^{v_{j}} \varphi_{j v}(\Theta) \varphi_{j v}(\Phi)\right),
\end{aligned}
$$

where $|x| \neq|y|$ and $\chi^{\prime}(1)=\left.w\left(Q_{1}(r), P_{1}(r)\right)\right|_{r=1}$ is its Wronskian. The series converges uniformly if either $|x| \leq k|y|$ or $|y| \leq k|x|$, where $0<k<1$.

For a nonnegative integer $m$ and two points $x, y \in H$, we define a modified Green function:

$$
G(b, m)(x, y)=G_{b}(x, y)-V(b, m)(x, y),
$$

where

$$
\begin{aligned}
& V(b, m)(x, y) \\
& = \begin{cases}\sum_{j=0}^{m} \frac{1}{\chi^{\prime}(1)} P_{j}(|x|) Q_{j}(|y|) & \text { if }|y|<1, \\
\quad \times\left(\sum_{v=1}^{v_{j}} \varphi_{j v}(\Theta) \varphi_{j v}(\Phi)\right) & \text { if } 1 \leq|y|<\infty .\end{cases}
\end{aligned}
$$

If the generalized Poisson kernel $P(b, m)\left(x, y^{\prime}\right)$ with respect to $H$ is defined by

$$
P(b, m)\left(x, y^{\prime}\right)=\frac{\partial G(b, m)(x, y)}{\partial n\left(y^{\prime}\right)},
$$

then we have $P(b, 0)\left(x, y^{\prime}\right)=P_{b}\left(x, y^{\prime}\right)$ and $P(0, m)\left(x, y^{\prime}\right)$ coincides with ones in Finkelstein and Scheinberg [7], Siegel and Talvila [8], Deng [9], Qiao and Deng [10], and Qiao [11] (see [5, Chapter 11]).

Put

$$
U(b, m ; u)(x)=\int_{\partial H} P(b, m)\left(x, y^{\prime}\right) u\left(y^{\prime}\right) d y^{\prime}
$$

where $u\left(y^{\prime}\right)$ is a continuous function on $\partial H$.

For real numbers $\beta \geq 1$, we denote $\mathscr{C}(\beta, b)$ the class of all measurable functions $f(y)$ satisfying the following inequality:

$$
\int_{H} \frac{|f(y)| \varphi_{1}}{1+P_{[\beta]}(|y|)|y|^{n+\{\beta\}}} d y<\infty,
$$

and the class $\mathscr{D}(\beta, b)$ consists of all measurable functions $g\left(y^{\prime}\right)\left(y^{\prime} \in \partial H\right)$ satisfying

$$
\int_{\partial H} \frac{\left|g\left(y^{\prime}\right)\right| P_{1}\left(\left|y^{\prime}\right|\right) Q_{1}\left(\left|y^{\prime}\right|\right)}{1+\chi^{\prime}\left(\left|y^{\prime}\right|\right) P_{[\beta]}\left(\left|y^{\prime}\right|\right)\left|y^{\prime}\right|^{n+\{\beta\}-1}} \frac{\partial \varphi_{1}}{\partial n} d y^{\prime}<\infty .
$$

We will also consider the class of all continuous functions $h(y)$, which is the solution of $(2)$, with $h^{+}(y) \in \mathscr{C}(\beta, b)$ and $h^{+}\left(y^{\prime}\right) \in \mathscr{D}(\beta, b)$, is denoted by $\mathscr{E}(\beta, b)$. 
Remark 2. If $b=0$ and $\beta=\alpha+1$, then (14) and (15) are equivalent to

$$
\begin{aligned}
& \int_{H} \frac{y_{n}|f(y)|}{1+|y|^{n+\alpha+2}} d y<\infty, \\
& \int_{\partial H} \frac{\left|g\left(y^{\prime}\right)\right|}{1+\left|y^{\prime}\right|^{n+\alpha}} d y^{\prime}<\infty
\end{aligned}
$$

respectively, from Remark 1 and (14), which yield that $\mathscr{E}(\alpha+$ $1,0)$ is equivalent to $(\mathrm{CH})_{\alpha}$ in the notation of [9].

Let us recall the classical case $b=0$. If $h(x) \geq 0$ is harmonic in $H$, continuous on $\bar{H}$, and $h \in \mathscr{E}(1,0)$, then there exists a constant $d_{3} \geq 0$ such that (see [12])

$$
h(x)=d_{3} x_{n}+\int_{\partial H} P\left(x, y^{\prime}\right) u\left(y^{\prime}\right) d y^{\prime},
$$

where $x=\left(x^{\prime}, x_{n}\right) \in H$.

Deng (see [9]) has constructed a similar representation to (17) for $h \in \mathscr{E}(\alpha+1,0)(\alpha \geq 0)$, which is the integral with a modified Poisson kernel derived by subtracting some special harmonic polynomials from $P\left(x, y^{\prime}\right)$. By virtue of this modified Poisson kernel, Qiao (see $[11,13])$ and Qiao and Deng (see [14-18]) have constructed different integral representations for harmonic functions of finite order and infinite order.

Especially, Su (see $[6,19])$ recently writes solutions to the half-space Dirichlet problem with respect to the stationary $S c h r o ̈ d i n g e r$ operator $S S E_{b}$. Now we state our main results as follows.

Theorem 3. If $h \in \mathscr{E}(\beta, b)$, then $h \in \mathscr{D}(\beta, b)$.

Theorem 4. If $h \in \mathscr{E}(\beta, b)$, $m$ is an integer such that $P_{m}(|y|)<$ $P_{[\beta]}(|y|)|y|^{\{\beta\}} \leq P_{m+1}(|y|)(|y| \geq 1)$, and then the following properties hold.

(I) If $\beta=1$, then there exists a constant $d_{4}$ such that

$$
h(x)=d_{4} P_{1}(|x|) \varphi_{1}(\Theta)+U(b, 0 ; h)(x)
$$

for $x \in H$.

(II) If $\beta>1$, then we have $h(x)=U(b, m ; h)(x)+u(x)$, where

$$
u(x)=\sum_{j=0}^{m}\left(\sum_{v=1}^{v_{j}} d_{j v} \varphi_{j v}(\Theta)\right) P_{j}(|x|)
$$

vanishing continuously on $\partial H, x=\left(x^{\prime}, x_{n}\right) \in H$ and $d_{j v}$ are constants.

\section{Lemmas}

The following Lemma plays an important role in our discussions, which is due to Levin and Kheyfits (see [5, page 356]).
Lemma 5. If $R>1$ and $h(y)$ is a solution of (2) on a domain containing $H(1, R)$, then

$$
\begin{aligned}
\int_{H R} & \frac{\chi^{\prime}(R)}{P_{1}(R)} h \varphi_{1}(\Phi) d S_{R}+\int_{\partial H(1, R)} h\left(y^{\prime}\right) \frac{\partial \varphi_{1}}{\partial n} W\left(\left|y^{\prime}\right|\right) d y^{\prime} \\
& +d_{5}+d_{6} \frac{Q_{1}(R)}{P_{1}(R)}=0
\end{aligned}
$$

where

$$
\begin{gathered}
W\left(\left|y^{\prime}\right|\right)=Q_{1}\left(\left|y^{\prime}\right|\right)-\frac{Q_{1}(R)}{P_{1}(R)} P_{1}\left(\left|y^{\prime}\right|\right), \\
d_{5}=\int_{H 1} h \varphi_{1}(\Phi) Q_{1}^{\prime}(r)-Q_{1}(1) \varphi_{1}(\Phi) \frac{\partial h}{\partial n} d S_{1}, \\
d_{6}=\int_{H 1} P_{1}(1) \varphi_{1}(\Phi) \frac{\partial h}{\partial n}-h \varphi_{1}(\Phi) P_{1}^{\prime}(1) d S_{1} .
\end{gathered}
$$

Lemma 6 (see [6, Corollary 1.6]). If $u$ is a continuous function on $\partial H$ satisfying

$$
\int_{\partial H} \frac{\left|u\left(y^{\prime}\right)\right|}{1+\left|y^{\prime}\right|^{\lambda_{m+1, s}^{+}+n-1}} d y^{\prime}<\infty
$$

then $U(b, m ; u)(x)$ is a solution of the Dirichlet problem for $S S E_{b}$ on $H$ with $h$ and

$$
\lim _{|x| \rightarrow \infty, x \in H}|x|^{-\lambda_{m+1, s}^{+}} U(b, m ; u)(x)=0
$$

Lemma 7 (see [6, Lemma 2.1] or [20, Theorem 1]). If $u(x)$ is a solution of (2) on $H$ satisfying

$$
\lim _{|x| \rightarrow \infty, x \in H}|x|^{-\lambda_{m+1, s}^{+}} u^{+}(x)=0,
$$

then (19) holds.

\section{Proof of Theorem 3}

We apply the formula $(20)$ to $h=h^{+}-h^{-}$in $H(1, R)$ :

$$
\begin{gathered}
m_{+}(R)+\int_{\partial H(1, R)} h^{+}\left(y^{\prime}\right) W\left(\left|y^{\prime}\right|\right) \frac{\partial \varphi_{1}}{\partial n} d y^{\prime}+d_{5}+\frac{Q_{1}(R)}{P_{1}(R)} d_{6} \\
=m_{-}(R)+\int_{\partial H(1, R)} h^{-}\left(y^{\prime}\right) W\left(\left|y^{\prime}\right|\right) \frac{\partial \varphi_{1}}{\partial n} d y^{\prime},
\end{gathered}
$$

where

$$
m_{ \pm}(R)=\int_{H R} \frac{\chi^{\prime}(R)}{P_{1}(R)} h^{ \pm} \varphi_{1} d S_{R} .
$$


Since $u \in \mathscr{E}(\beta, b)$, we obtain by (8)

$$
\begin{aligned}
& \int_{1}^{\infty} \frac{m_{+}(R) P_{1}(R)}{\chi^{\prime}(R) P_{[\beta]}(R) R^{n+\{\beta\}}} d R \\
& \quad=\int_{H(1, \infty)} \frac{h^{+} \varphi_{1}}{P_{[\beta]}(|y|)|y|^{n+\{\beta\}}} d y \\
& \quad \leq 2 \int_{H} \frac{h^{+} \varphi_{1}}{1+P_{[\beta]}(|y|)|y|^{n+\{\beta\}}} d y<\infty .
\end{aligned}
$$

From (15), we conclude that

$$
\begin{aligned}
\int_{1}^{\infty} & \frac{P_{1}(R)}{\chi^{\prime}(R) P_{[\beta]}(R) R^{n+\{\beta\}}} \\
& \times \int_{\partial H(1, R)} h^{+}\left(y^{\prime}\right) W\left(\left|y^{\prime}\right|\right) \frac{\partial \varphi_{1}}{\partial n} d y^{\prime} d R \\
= & \int_{\partial H(1, \infty)} h^{+}\left(y^{\prime}\right) P_{1}\left(\left|y^{\prime}\right|\right) \\
& \times \int_{\left|y^{\prime}\right|}^{\infty} \frac{P_{1}(R)}{\chi^{\prime}(R) P_{[\beta]}(R) R^{n+\{\beta\}}} \\
\leq & \left.M \int_{\partial H(1, \infty)} \frac{\left(Q_{1}\left(\left|y^{\prime}\right|\right)\right.}{\chi_{1}\left(\left|y^{\prime}\right|\right)}-\frac{W_{1}(R)}{P_{1}(R)}\right) d R \frac{\partial \varphi_{1}}{\partial n} d y^{\prime} \\
\leq & M \int_{\partial H} \frac{h^{+}\left(y^{\prime}\right) P_{1}\left(\left|y^{\prime}\right|\right) Q_{[}\left(\left|y^{\prime}\right|\right)}{1+\chi^{\prime}\left(\left|y^{\prime}\right|\right)\left|y^{\prime}\right|^{n+\{\beta\}-1} \frac{\partial \varphi_{1}}{\partial n} d y^{\prime}} \\
<\infty & \infty P_{1 \beta}\left(\left|y^{\prime}\right|\right) Q_{1}\left(\left|y^{\prime}\right|\right) \\
&
\end{aligned}
$$

Combining (25), (27), and (28), we obtain

$$
\begin{aligned}
\int_{1}^{\infty} & \frac{P_{1}(R)}{\chi^{\prime}(R) P_{[\beta]}(R) R^{n+(\{\beta\} / 2)}} \\
& \times \int_{\partial H(1, R)} h^{-}\left(y^{\prime}\right) W\left(\left|y^{\prime}\right|\right) \frac{\partial \varphi_{1}}{\partial n} d y^{\prime} d R \\
\leq & \int_{1}^{\infty} \frac{m_{+}(R) P_{1}(R)}{\chi^{\prime}(R) P_{[\beta]}(R) R^{n+(\{\beta\} / 2)}} d R \\
& +\int_{1}^{\infty} \frac{P_{1}(R)}{\chi^{\prime}(R) P_{[\beta]}(R) R^{n+(\{\beta\} / 2)}} \\
& \times \int_{\partial H(1, R)} h^{+}\left(y^{\prime}\right) W\left(\left|y^{\prime}\right|\right) \frac{\partial \varphi_{1}}{\partial n} d y^{\prime} d R \\
& +\int_{1}^{\infty} \frac{1}{\chi^{\prime}(R) P_{[\beta]}(R) R^{n+(\{\beta\} / 2)}} \\
& \times\left(P_{1}(R) d_{5}+Q_{1}(R) d_{6}\right) d R
\end{aligned}
$$

$$
<\infty
$$

Set

$$
\begin{aligned}
\mathscr{H}(\beta)= & \lim _{\left|y^{\prime}\right| \rightarrow \infty} \frac{\chi^{\prime}\left(\left|y^{\prime}\right|\right) P_{[\beta]}\left(\left|y^{\prime}\right|\right)\left|y^{\prime}\right|^{n+\{\beta\}-1}}{Q_{1}\left(\left|y^{\prime}\right|\right)} \\
& \times \int_{\left|y^{\prime}\right|}^{\infty} \frac{P_{1}(R)}{\chi^{\prime}(R) P_{[\beta]}(R) R^{n+(\{\beta\} / 2)}} \\
= & \times\left(\frac{Q_{1}\left(\left|y^{\prime}\right|\right)}{P_{1}\left(\left|y^{\prime}\right|\right)}-\frac{W_{1}(R)}{P_{1}(R)}\right) d R \\
& \quad \times \int_{\left|y^{\prime}\right| \rightarrow \infty}^{\infty} \frac{1}{\left.y^{\prime}\right|^{\lambda_{[\beta], s}^{+}+\lambda_{1, s}^{+}+n+\{\beta\}-2}} \\
& \quad R^{\lambda_{[\beta], s}^{+}-\lambda_{1, s}^{+}+(\{\beta\} / 2)+1}\left(\frac{1}{\left|y^{\prime}\right|^{\chi_{1, s}}}-\frac{1}{R^{\chi_{1, s}}}\right) d R
\end{aligned}
$$

where $\chi_{1, s}=\lambda_{1, s}^{+}-\lambda_{1, s}^{-}$.

By the L'hospital's rule, we have

$$
\begin{aligned}
& \mathscr{H}(\beta) \\
& = \begin{cases}\frac{\chi_{1, s}}{\left(\lambda_{[\beta], s}^{+}-\lambda_{1, s}^{+}\right)\left(\lambda_{[\beta], s}^{+}+\lambda_{1, s}^{+}+n-2\right)} & \text { if }\{\beta\}=0, \\
+\infty & \text { if }\{\beta\} \neq 0,\end{cases}
\end{aligned}
$$

which yields that there exists a positive constant $M$ such that, for any $\left|y^{\prime}\right| \geq 1$,

$$
\begin{gathered}
\int_{\left|y^{\prime}\right|}^{\infty} \frac{P_{1}(R)}{\chi^{\prime}(R) P_{[\beta]}(R) R^{n+(\{\beta\} / 2)}} W\left(\left|y^{\prime}\right|\right) d R \\
\geq \frac{M P_{1}\left(\left|y^{\prime}\right|\right) Q_{1}\left(\left|y^{\prime}\right|\right)}{\chi^{\prime}\left(\left|y^{\prime}\right|\right) P_{[\beta]}\left(\left|y^{\prime}\right|\right)\left|y^{\prime}\right|^{n+\{\beta\}-1}} .
\end{gathered}
$$

Then

$$
\begin{gathered}
M \int_{\partial H(1, \infty)} \frac{h^{-}\left(y^{\prime}\right) P_{1}\left(\left|y^{\prime}\right|\right) Q_{1}\left(\left|y^{\prime}\right|\right)}{\chi^{\prime}\left(\left|y^{\prime}\right|\right) P_{[\beta]}\left(\left|y^{\prime}\right|\right)\left|y^{\prime}\right|^{n+\{\beta\}-1}} \frac{\partial \varphi_{1}}{\partial n} d y^{\prime} \\
\leq \int_{\partial H(1, \infty)} h^{-} \int_{\left|y^{\prime}\right|}^{\infty} \frac{P_{1}(R)}{\chi^{\prime}(R) P_{[\beta]}(R) R^{n+(\{\beta\} / 2)}} \\
\times W\left(\left|y^{\prime}\right|\right) d R \frac{\partial \varphi_{1}}{\partial n} d y^{\prime} \\
\quad<\infty,
\end{gathered}
$$

which shows that $h \in \mathscr{D}(\beta, b)$ from $|h|=h^{+}+h^{-}$. Then Theorem 3 is proved.

\section{Proof of Theorem 4}

To prove (II), notice that $P_{m}(|y|)<P_{[\beta]}(|y|)|y|^{\{\beta\}} \leq$ $P_{m+1}(|y|)(|y| \geq 1)$ and condition (15) is stronger than condition (22) from Theorem 3. 
Consider the function $h(x)-U(a, m ; h)(x)$. Then it follows from Lemma 6 and Theorem 3 that this is a solution of (2) in $H$ and vanishes continuously on $\partial H$.

Then

$$
0 \leq(h(x)-U(b, m ; h)(x))^{+} \leq h^{+}(x)+(U(b, m ; h))^{-}(x)
$$

for any $P \in H$. Further, (8) gives that

$$
\lim _{|x| \rightarrow \infty, x \in H}|x|^{-\lambda_{m+1, s}^{+}} h^{+}(x)=0,
$$

which together with (34) and Lemmas 6 and 7 give the result of (II).

If $u \in \mathscr{E}(1, b)$, then $h \in \mathscr{E}(\beta, b)$ for each $\beta>1$ and there exists a constant $d_{7}$ such that

$$
h(x)=d_{7} P_{1}(|x|) \varphi_{1}(\Theta)+U(b, 1 ; h)(x)
$$

for all $x \in H$. So if we take

$$
d_{4}=d_{7}-\int_{\partial H[1, \infty)} P(b, 1)\left(0, y^{\prime}\right) h\left(y^{\prime}\right) d y^{\prime},
$$

we see that (18) holds for all $x \in H$.

Thus we complete the proof of Theorem 4 .

\section{Acknowledgments}

The author wishes to expresseses his appreciation to Professor Jingben Yin for very useful conversations related to this problem. He is grateful to the referee for her or his careful reading and helpful suggestions which led to the improvement of the original paper. This work is supported by the National Natural Science Foundation of China under Grant 11226093.

\section{References}

[1] M. Reed and B. Simon, Methods of Modern Mathematical Physics, vol. 3, Academic Press, London, UK, 1970.

[2] G. Rosenblum, M. Solomyak, and M. Shubin, Spectral Theory of Differential Operators, VINITI, Moscow, Russia, 1989.

[3] B. Simon, "Schrödinger semigroups," Bulletin of the American Mathematical Society, vol. 7, no. 3, pp. 447-526, 1982.

[4] P. Hartman, Ordinary Differential Equations, John Wiley \& Sons Inc., New York, NY, USA, 1964.

[5] B. Y. Levin and A. I. Kheyfits, "Asymptotic behavior of subfunctions of time-independent Schrödinger operators," in Some Topics on Value Distribution and Differentiability in Complex and P-Adic Analysis, vol. 11, pp. 323-397, Science Press, Beijing, China, 2008.

[6] B. Y. Su, "Dirichlet Problem for the Schrödinger operator in a half space," Abstract and Applied Analysis, vol. 2012, Article ID 578197, 14 pages, 2012.

[7] M. Finkelstein and S. Scheinberg, "Kernels for solving problems of Dirichlet type in a half-plane," Advances in Mathematics, vol. 18, no. 1, pp. 108-113, 1975.

[8] D. Siegel and E. Talvila, "Sharp growth estimates for modified Poisson integrals in a half space," Potential Analysis, vol. 15, no. 4, pp. 333-360, 2001.
[9] G. T. Deng, "Integral representations of harmonic functions in half spaces," Bulletin des Sciences Mathématiques, vol. 131, no. 1, pp. 53-59, 2007.

[10] L. Qiao and G. T. Deng, "Growth estimates for modified Green potentials in the upper-half space," Bulletin des Sciences Mathématiques, vol. 135, no. 3, pp. 279-290, 2011.

[11] L. Qiao, "Integral representations for harmonic functions of infinite order in a cone," Results in Mathematics, vol. 61, no. 12, pp. 63-74, 2012.

[12] E. M. Stein, Singular Integrals and Differentiability Properties of Functions, Princeton University Press, Princeton, NJ, USA, 1970.

[13] L. Qiao, "Modified Poisson integral and Green potential on a half-space," Abstract and Applied Analysis, vol. 2012, Article ID 765965, 13 pages, 2012.

[14] L. Qiao and G. T. Deng, “The Riesz decomposition theorem for superharmonic functions in a cone and its application," Scientia Sinica Mathematica, vol. 42, no. 8, pp. 763-774, 2012 (Chinese).

[15] L. Qiao and G. T. Deng, "Integral representations of harmonic functions in a cone," Scientia Sinica Mathematica, vol. 41, no. 6, pp. 535-546, 2011 (Chinese).

[16] L. Qiao and G. T. Deng, "Growth estimate for a class of harmonic functions in a cone," Acta Mathematica Sinica, vol. 54, no. 6, pp. 1021-1028, 2011 (Chinese).

[17] L. Qiao and G. T. Deng, "Growth property and integral representation of harmonic functions in a cone," Bulletin of the Malaysian Mathematical Sciences Society, vol. 36, no. 2, pp. 511$523,2013$.

[18] L. Qiao and G. T. Deng, "Integral representation for the solution of the stationary Schrödinger equation in a cone," Mathematische Nachrichten, vol. 285, no. 16, pp. 2029-2038, 2012.

[19] B. Y. Su, "Growth properties of harmonic functions in the upper half space," Acta Mathematica Sinica, vol. 55, no. 6, pp. 10951100, 2012 (Chinese).

[20] A. I. Kheyfits, "Liouville theorems for generalized harmonic functions," Potential Analysis, vol. 16, no. 1, pp. 93-101, 2002. 


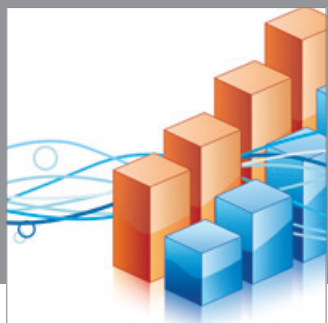

Advances in

Operations Research

mansans

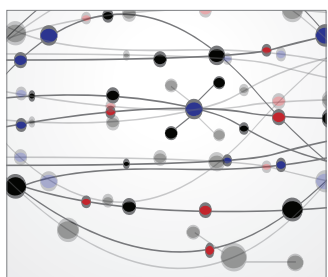

The Scientific World Journal
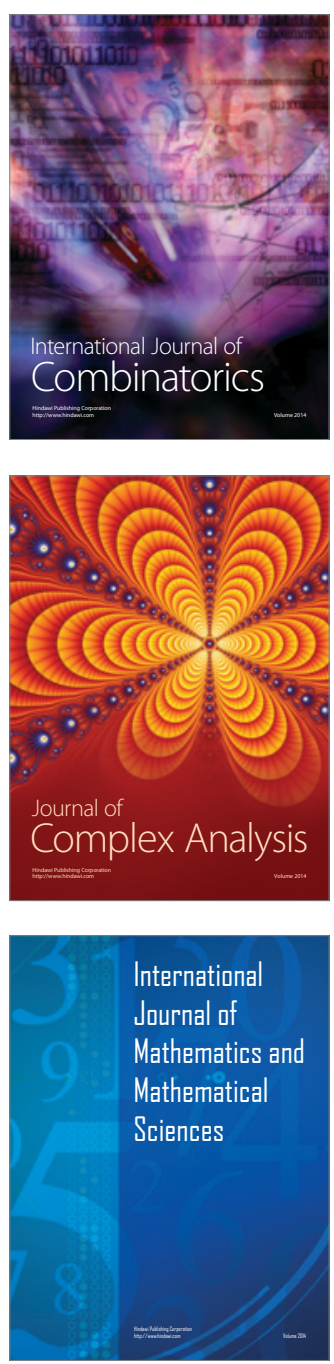
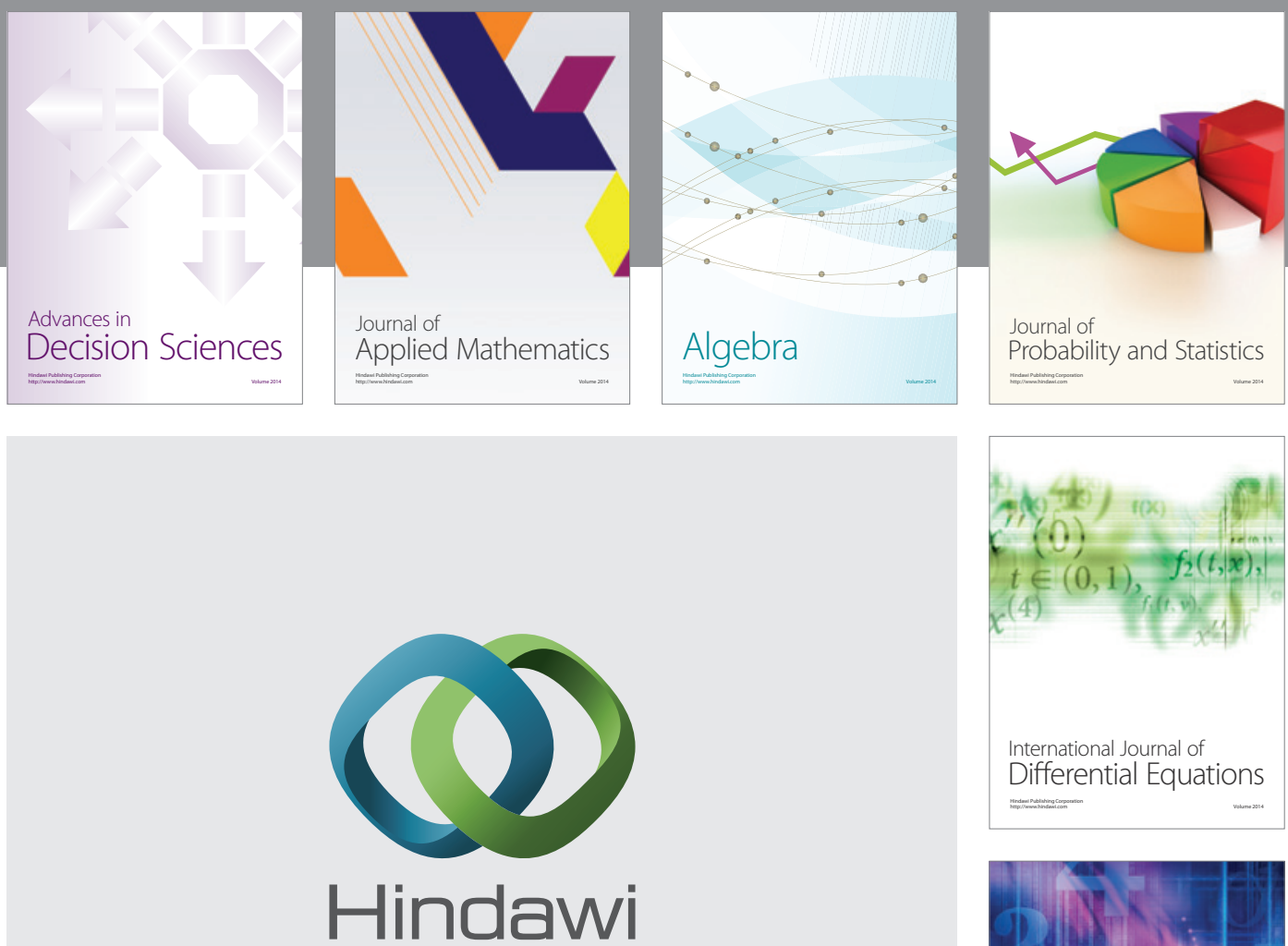

Submit your manuscripts at http://www.hindawi.com
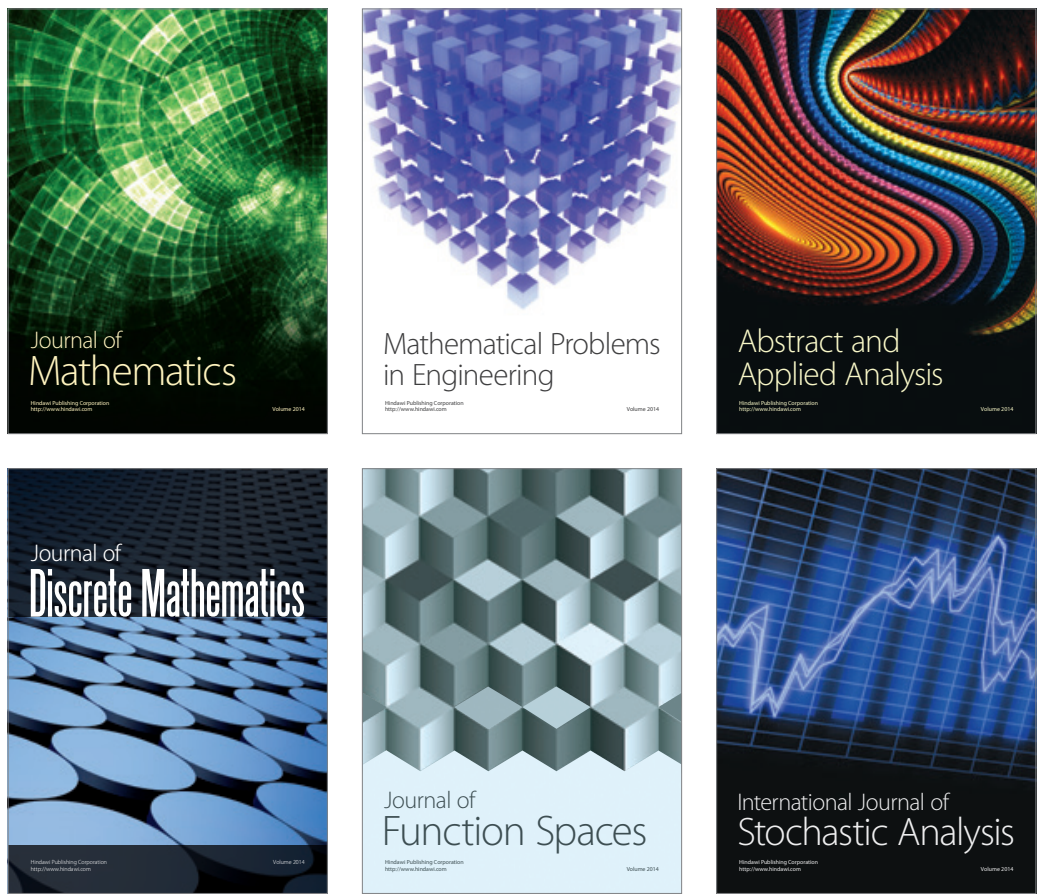

Journal of

Function Spaces

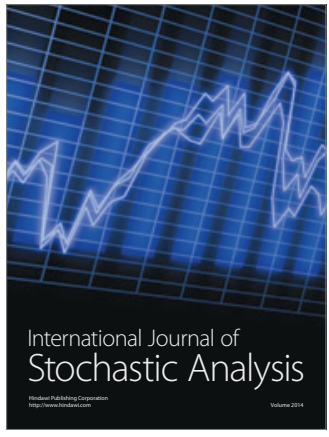

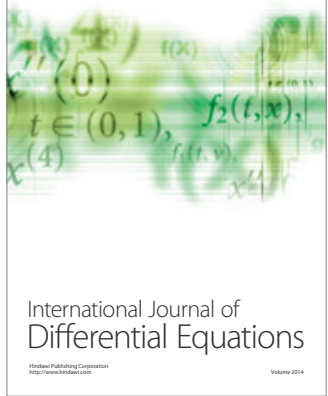
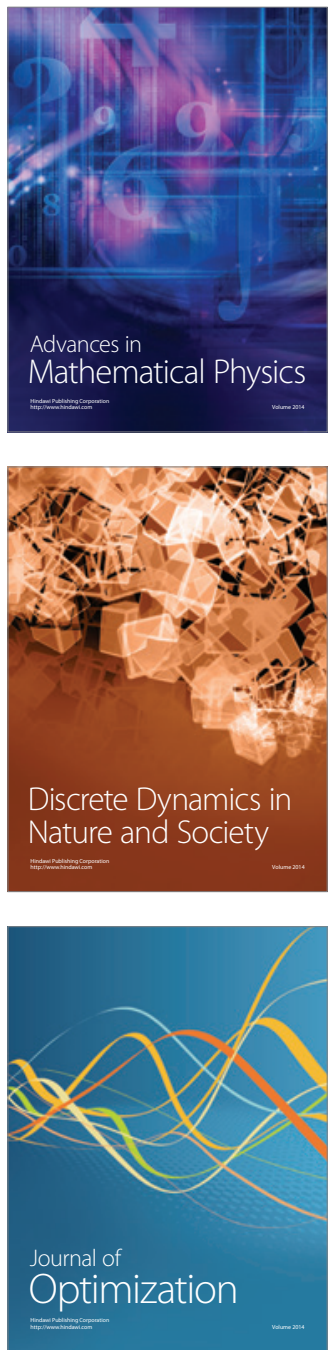